\title{
Assessing introspective awareness of attention capture
}

\section{Owen J. Adams ${ }^{1} \cdot$ Nicholas Gaspelin ${ }^{1}$}

Published online: 22 January 2020

(C) The Psychonomic Society, Inc. 2020

\begin{abstract}
Visual attention can sometimes be involuntarily captured by salient stimuli, and this may lead to impaired performance in a variety of real-world tasks. If observers were aware that their attention was being captured, they might be able to exert control and avoid subsequent distraction. However, it is unknown whether observers can detect attention capture when it occurs. In the current study, participants searched for a target shape and attempted to ignore a salient color distractor. On a subset of trials, participants then immediately classified whether the salient distractor captured their attention ("capture" vs. "no capture"). Participants were slower and less accurate at detecting the target on trials on which they reported "capture" than "no capture." Follow-up experiments revealed that participants specifically detected covert shifts of attention to the salient item. Altogether, these results indicate that observers can have immediate awareness of visual distraction, at least under certain circumstances.
\end{abstract}

Keywords Attentional capture · Visual search · Visual awareness

Physically salient stimuli, such as brightly colored objects or blinking lights, seem to involuntarily capture our visual attention. For example, if we are intently searching a grocery store aisle for a green box of cereal, we might be automatically captured by a bright yellow sale sign, even though it does not match our immediate goals. Automatic distraction by salient stimuli (called attention capture) has been a hotbed of research for the past several decades. This is because understanding attention capture is a crucial step toward developing basic science models of visual search (Wolfe \& Horowitz, 2017) and is relevant to any applied setting where a visual warning signal is used to indicate potential hazards (Gaspelin \& Luck, 2019).

One common paradigm used to investigate attention capture is the additional singleton paradigm (Theeuwes, 1992). In this task, participants search for a unique shape (e.g., a diamond) among homogenously shaped distractors (e.g., circles). On some trials, one distractor item is a color singleton (e.g., a pink item amongst green items). This salient distractor is never the target item, and therefore, should be ignored. Nevertheless, response times (RTs) are typically slower on singleton-present trials than singleton-absent trials. This singleton-presence cost was initially taken as evidence for

Owen J. Adams

oadams2@binghamton.edu

1 Department of Psychology, Binghamton University, State University of New York, P.O. Box 6000, Binghamton, NY 13902-6000, USA stimulus-driven models, which propose that the salient item automatically captures attention, slowing detection of the search target.

More recent research has shown that, under conditions that encourage strong top-down control, capture by physically salient items can be avoided (Bacon \& Egeth, 1994; Folk, Leber, \& Egeth, 2002; Folk, Remington, \& Johnston, 1992). More specifically, it is now clear that observers can learn to proactively inhibit salient objects in order to prevent attention capture (Cunningham \& Egeth, 2016; Gaspar \& McDonald, 2014, 2018; Gaspelin, Leonard, \& Luck, 2015, 2017; Gaspelin \& Luck, 2018a, 2018b, 2018c; Stilwell, Bahle, \& Vecera, 2019; Vatterott \& Vecera, 2012; Won \& Geng, 2018). A key question, however, is exactly how individuals learn to do this, especially in real-world situations without direct feedback about performance. One possibility is that individuals have introspective awareness of visual distraction (Flavell, 1979; Nisbett \& Wilson, 1977). That is, individuals may be able to detect when they were momentarily captured via internal cognitive monitoring. When capture is detected, they might then exert top-down control to avoid subsequent distraction. Consistent with this claim, in the metacognitive literature, the ability to cognitively monitor performance is well established to be a crucial step toward error correction (Fletcher \& Carruthers, 2012). However, it is currently unknown whether individuals even possess the ability to internally detect attention capture when it occurs.

There are some reasons to suspect that introspective awareness of attention capture may be lacking. The visual system 


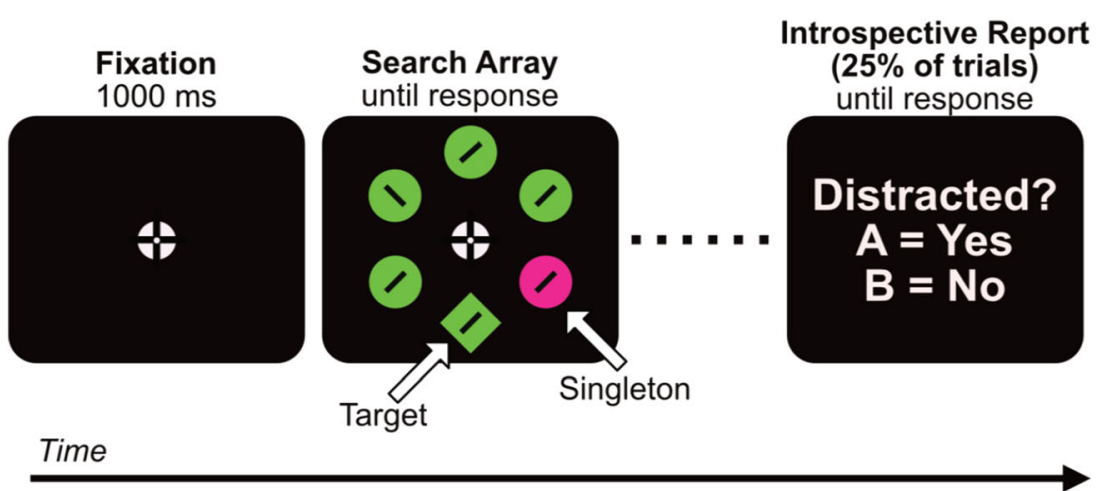

Fig. 1 The experimental paradigm from Experiment 1. On search-only trials (75\% of trials), participants searched for a target shape (e.g., diamond) amongst homogenous distractor shapes (e.g., circles) and indicated the angle of a line inside via manual button press. On half of trials, a color singleton distractor was presented at a nontarget location. On introspective-report trials (25\% of trials), participants performed the same search task, but afterward reported whether or not they were captured by the salient distractor. Singletons were always present on introspectivereport trials reorients several times per second during search, whether via overt eye movements (Henderson, 2003) or covert shifts of attention (Horowitz, Wolfe, Alvarez, Cohen, \& Kuzmova, 2009). Successive attentional shifts could happen so quickly $(<150 \mathrm{~ms})$ that observers may simply fail to notice that they were momentarily captured by a salient item. Furthermore, individuals may not typically monitor where attention is directed during visual search and therefore, may have a general inability to recall where attention was allocated (called inattentional amnesia; Vö, Aizenman, \& Wolfe, 2016; Wolfe, 1999). Finally, there is now an abundance of evidence that visual attention is guided, in a seemingly unconscious manner, by recent experience (Anderson, 2013; Anderson \& Kim, 2018; Anderson, Laurent, \& Yantis, 2011; Awh, Belopolsky, \& Theeuwes, 2012). If participants lack awareness of the factors that guide attention, then they may also lack awareness of where attention was directed.

As far as we know, no studies have directly assessed whether participants have immediate awareness of attention capture. However, some relevant studies have investigated related questions. For example, Lamy, Alon, Carmel, and Shalev (2015) assessed whether salient cues below the threshold of conscious awareness could capture attention. Importantly, these subliminal cues captured attention, even when observers reported that they did not consciously perceive the cue (see also Mulckhuyse, Talsma, \& Theeuwes, 2007). This study suggests that participants were captured even though they were unaware that a salient item was present. However, it did not answer whether they were aware that their attention shifted to the salient item. Also, because these studies were concerned with subliminal salient cues, they used experimental techniques and stimuli that are very different from those typically used to study attention capture.

Other studies have used a different approach to assess metacognitive awareness of capture: Participants report their general distractibility via questionnaires (Burra \& Kerzel, 2014; Proulx, 2011). Responses on these questionnaires were then compared with performance on an attention capture task. Typically, the questionnaire responses were not indicative of performance on the capture task. For example, Proulx (2011) found capture by a salient item for 34 of 40 participants, despite all participants reporting the salient item was "not distracting." However, this should not be taken as definitive evidence that participants completely lack the ability to detect visual distraction. Under ordinary circumstances, individuals may not keep track of where attention is allocated during visual search (Vö et al., 2016; Wolfe, 1999). As a result, if participants are not informed that they should monitor performance, they may be unable to recollect their overall distraction level after completing an attention capture task. Furthermore, there is strong evidence that metacognitive accuracy is impaired with long delays between the judgments of performance and the performance itself (Fleming \& Lau, 2014). Simply put, participants may have the ability to detect visual distraction, but their awareness may be fleeting and may not occur unless they are explicitly encouraged to cognitively monitor performance.

In summary, understanding if participants can internally monitor visual distraction is a crucial step to understanding how observers learn to avoid visual distraction in real-world tasks. However, it is currently unknown if participants have any ability to detect attention capture. In the current study, we used a novel technique that directly assessed awareness of attention capture by having participants immediately classify whether they were captured after completing a trial of an attention capture task. This allows us to avoid issues associated with delayed assessment of cognitive performance that have been highlighted in the recent literature on metacognition (Fleming \& Lau, 2014). 


\section{Experiment 1}

Participants performed a modified additional singleton paradigm (see Fig. 1). On search-only trials, participants searched for a target shape (e.g., diamond) among a homogenous set of distractor shapes (e.g., circles) and reported the tilt of a line inside. On half of trials, a salient distractor appeared at a nontarget location. If the singleton captures attention, response time should be slower on singleton-present trials than singleton-absent trials. Previous studies have shown that this type of task strongly encourages attention capture by the singleton, because participants search broadly for any uniquely featured item (singleton detection mode; Bacon \& Egeth, 1994; Gaspelin et al., 2015; Pashler, 1988).

Importantly, on a subset of trials, participants performed an introspective-report task. Participants first located a target shape and attempted to ignore a salient distractor (just as on search-only trials). They were given no direct feedback about their performance on the search task. Next, they were asked to classify whether the salient singleton captured their attention on that trial ("capture" or "no capture"). If participants were aware of whether they were captured on a given trial, search performance should be slower and less accurate on "capture" report trials than "no capture" report trials. If participants were unaware of capture, however, search performance should be equivalent on "capture" report trials and "no capture" report trials.

\section{Method}

Participants An a priori sample size of 32 participants was chosen on the basis of previous attention capture experiments using similar paradigms (Bacon \& Egeth, 1994; Gaspelin et al., 2015; Theeuwes, 1992). If we assume the difference between "capture" report trials and "no capture" report trials is similar in magnitude to a singleton-presence cost (Gaspelin et al., 2015), the current sample size would yield $>.999$ power to detect an effect. However, the magnitude of corresponding differences between "capture" report trials and "no capture" report trials is unknown given that the introspective-report paradigm is entirely new. Thus, we collected large sample sizes to ensure sufficient statistical power for detecting effects of introspective reports. We also replicate the basic pattern of results in Experiment 2.

Participants were undergraduate students from Binghamton University, State University of New York, who volunteered for course credit. One participant was replaced for having a manual error rate of more than 2.5 standard deviations from the group mean $(>20 \%)$. Two participants were replaced for reporting "no capture" on more than $70 \%$ of introspective-report trials (which yielded insufficient power to perform the introspective-report analysis). Of the final sample of 32 participants, 25 were women and seven were men.
The mean age was 18.8 years. All participants had normal color vision, as assessed by an Ishihara color vision test, as well as normal or corrected-to-normal visual acuity.

Apparatus Stimuli were presented using PsychToolbox (Brainard, 1997) for MATLAB on an Asus VS248H-P LCD monitor with a black background, placed at a viewing distance of $100 \mathrm{~cm}$. A photosensor was used to measure the timing delay of the video system (12 ms), and this delay was subtracted from all latency values reported in this paper.

Stimuli and procedure Each search display consisted of six shapes distributed at equal distances around a notional circle with a radius of $3.0^{\circ}$, measured to the center of each shape (see Fig. 1). The shapes were circles $\left(0.9^{\circ}\right.$ in diameter) and diamonds $\left(1.1^{\circ}\right.$ in diameter) drawn in photometrically isoluminant colors: green $\left(26.9 \mathrm{~cd} / \mathrm{m}^{2}, x=.28, y=.62\right)$ or pink $\left(26.9 \mathrm{~cd} / \mathrm{m}^{2}\right.$, $x=.53, y=.27$ ). Each shape contained a black line that subtended $0.6^{\circ} \times 0.1^{\circ}$ and was tilted $45^{\circ}$ to the left or right. A gray fixation cross $\left(0.3^{\circ} \times 0.3^{\circ}\right)$ was visible throughout the trial, except that it was absent during the introspective reports.

Each search display consisted of a target shape (e.g., a diamond) and five homogenous distractors (i.e., circles among diamonds for half of participants and diamonds among circles for the other half). Participants searched for the unique shape and made a manual button press to classify the tilt of a black line inside. On half of trials, all items were the same color (singleton absent). On remaining trials, one distractor item was a different color than the other search items (singleton present). The location of the singleton distractor was selected at random on each trial, with the exception that it was never presented at the target location. The target location was also selected at random. Given that the singleton never appeared at the target location, participants were instructed to ignore the singleton. Previous studies have shown that this type of task strongly encourages attention capture by the singleton, because participants search broadly for any uniquely featured item (singleton detection mode; Bacon \& Egeth, 1994; Gaspelin et al., 2015; Pashler, 1988).

There were two types of trials, which were randomly intermixed throughout the experimental session. On searchonly trials ( $75 \%$ of overall trials), participants searched for the target shape and made a speeded manual button press regarding the tilt of the line inside (left or right) using a game pad (using the left or right trigger, respectively). On introspective-report trials ( $25 \%$ of overall trials), participants performed the same search task, with the exception that a color singleton distractor was always present on these trials. Afterward, participants were asked whether or not they were captured by the singleton on that trial. A screen appeared with the word "Distracted?" and participants used the game pad to report "yes" or "no" (using the "A" button and "B" button, respectively). At the beginning of each experimental session, participants received written and 
verbal instructions that explained how to use this prompt. Specifically, participants were instructed to report whether or not their visual attention was allocated to the uniquely colored item. The singleton distractor was present on half of all trials. On half of singleton-present trials, we included an introspective-report task. Therefore, the singleton was present on one-third $(33.3 \%)$ of search-only trials, but was present on all $(100 \%)$ of introspective-report trials.

Search-only trials started by presenting the fixation cross for 1,000 ms. After fixation, the search array was presented until a response was made. On introspective-report trials, the search response was followed by a prompt where the participant reported whether or not they were captured. The response to this prompt was not timed.

Participants first performed one practice block of 60 search-only trials. Next, they performed one practice block of search-only trials intermixed with introspective-report trials. Next, the participants performed eight blocks of 60 trials each (360 search-only trials and 120 introspective-report trials), leading to a total of 480 trials. On the initial practice block, participants received immediate performance feedback. If the participant took more than 2,000 ms to respond, a timeout display ("Too Slow") was shown for $500 \mathrm{~ms}$. If the participant responded incorrectly, a $200-\mathrm{Hz}$ tone was presented for $500 \mathrm{~ms}$. After this first block, participants did not receive any feedback about performance. Therefore, any RT differences observed on introspective-report trials could not be due to experimenter feedback.

At the end of each block, participants received feedback on mean RT and accuracy. During these block breaks, they were warned if their accuracy was below $90 \%$ or if they chose the same answer to the distraction question on more than $70 \%$ of trials (i.e., to discourage participants from always responding "yes" or "no").

After the last block break, participants were given a survey to assess their self-perceived level of distraction by the color singleton ("Was the uniquely colored item distracting?"). They could choose one of five options on a scale ranging from 1 (not at all) to 5 (very distracting). We administered these postexperiment questionnaires for comparison with previous studies assessing delayed introspective awareness of attention capture (Burra \& Kerzel, 2014; Proulx, 2011). However, the aforementioned metacognition literature shows abundant evidence of metacognitive impairments with long delays between judgments of performance and actual performance (Fleming \& Lau, 2014; Maniscalco \& Lau, 2012). Therefore, we express caution in the interpretation of these post-experiment survey results.

\section{Results}

Trials with RTs less than $200 \mathrm{~ms}$ or greater than $1,500 \mathrm{~ms}$ were excluded from all analyses ( $0.7 \%$ of trials). Trials with incorrect responses $(3.3 \%)$ were omitted from RT analyses.
Search-only trials As shown in Fig. 2, responses were slower on singleton-present trials $(655 \mathrm{~ms})$ than singleton-absent trials (602 ms): a 53-ms singleton presence cost, $t(31)=8.48, p<$ $.001, d=1.50$. This difference indicates that visual attention was captured by the salient item, slowing detection of the target when it was present (Theeuwes, 1992). It is important to clarify that we specifically encouraged capture by the color singleton distractor by making the target a shape singleton. This experimental design is well-known to encourage participants to search for any uniquely featured item, allowing the singleton distractor to capture attention (singleton detection mode; Bacon \& Egeth, 1994). This aspect of the design is crucial. If participants were never captured by the color singleton, there may have been a floor effect in the subsequent analysis of introspective-report trials.

Participants did not commit many errors during this task. Error rates were not significantly higher on singleton-present trials $(3.5 \%)$ than on singleton-absent trials $(3.3 \%), t(31)=$ $0.74, p=.468, d=0.13$.

Introspective-report trials We next compared mean RT on trials where participants reported distraction by the singleton ("capture" trials) with trials where participants reported no distraction by the singleton ("no capture" trials). Recall that participants received no accuracy or RT feedback on introspective-report trials in this analysis. All time-out trials $(>2,000 \mathrm{~ms})$ have been excluded from the analyses. Therefore, any differences in mean RT must reflect some internalized detection of a cognitive error.

As can be seen in the right-hand panel of Fig. 2, responses were slower on "capture" trials $(690 \mathrm{~ms})$ than "no capture" trials $(627 \mathrm{~ms}), t(31)=6.78, p<.001, d=1.20$. This pattern of results is consistent with the interpretation that visual attention was misdirected to the color singleton on trials where participants reported "capture" and that this misdirection of

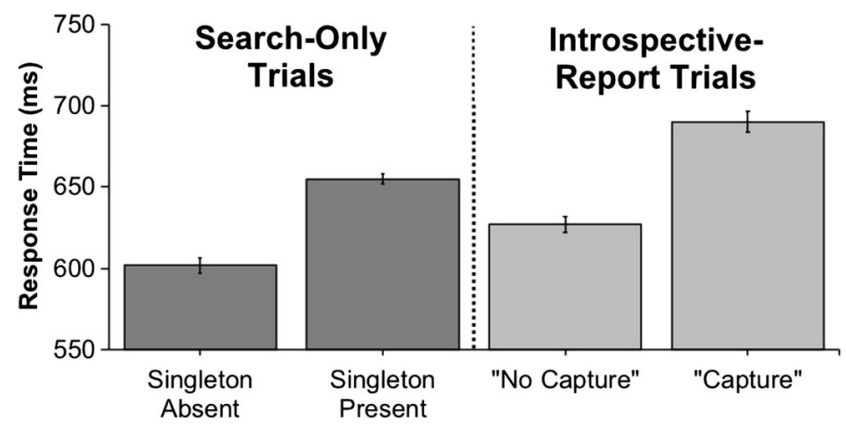

Fig. 2 Mean response times from Experiment 1 on search-only trials and introspective-report trials. The singleton was always present on introspective-report trials. On "no capture" trials, participants reported no distraction by the salient item on that trial, whereas on "capture" trials, participants reported distraction by the salient item on that trial. Error bars in all figures in this paper represent the within-subjects standard error of the mean (Cousineau, 2005; Morey, 2008) 
attention slowed detection of the target stimulus. In other words, this result clearly suggests that participants were immediately aware of capture by the singleton. In fact, of the 32 participants in this experiment, 28 showed the basic effect of slower RTs on "capture" report trials than "no capture" report trials.

If participants had perfect awareness of capture, they should never have been captured on trials where they reported "no capture." As a result, performance on "no capture" trials with a color singleton should have been functionally equivalent to trials where the singleton was absent. This would yield equally fast mean RTs on "no capture" report trials and singleton-absent trials. As shown in Fig. 2, RTs were significantly slower on "no capture" report trials $(627 \mathrm{~ms})$ than in singleton-absent trials $(602 \mathrm{~ms}), t(31)=5.19, p<.001, d=$ 0.92 . Therefore, it seems likely that participants were not able to classify the absence of attention capture with perfect accuracy.

We also compared error rates on "capture" report and "no capture" report trials. Interestingly, error rates were higher for "capture" trials $(6.1 \%)$ than "no capture" trials $(0.5 \%), t(31)=$ $8.37, p<.001, d=1.48$. This is consistent with the interpretation that participants temporarily misdirected attention to the singleton on "capture" report trials, which reduced accuracy at reporting the tilt of the line inside of the target stimulus. It is important to clarify that inaccurate trials were excluded from the previous RT analyses. Therefore, the previously observed RT differences cannot be explained by mere awareness of response accuracy.

Post-experiment surveys After the search task, participants performed a brief questionnaire where they estimated overall level of distraction by the salient item (1 meaning not distracting at all and 5 meaning very distracting). On average, participants reported 2.8, indicating that they felt an intermediate level of distraction from the color singleton. We then correlated each individual's scores on the final distraction survey with their singleton-presence costs on search-only trials (i.e., their RT-based index of attention capture). Participants who reported higher levels of distraction from the salient item on the post-experiment survey also showed significantly higher singleton-presence costs in search-only trials $(r=.43$, $p=.014)$. This result was surprising considering previous research (Burra \& Kerzel, 2014; Proulx, 2011) and the extensive literature on the failure of this approach to assess metacognitive awareness (Fleming \& Lau, 2014). As will be seen in Experiments 2 and 3, the questionnaire approach is much less robust and replicable than the immediate introspective-report approach.

We also correlated the percentage of "capture" report trials for each participant with responses on the post-experiment survey and singleton-presence costs. The percentage of "capture" report trials was uncorrelated with the magnitude of singleton-presence costs on search only trials $(r=.16, p=$ $.381)$, but was correlated with the post-experiment survey score $(r=.36, p=.044)$. An important caveat is that we explicitly encouraged participants to report "capture" and "no capture" on a roughly equal number of trials to ensure enough trials per analysis bin for calculating mean RT and accuracy. Therefore, these correlations should be interpreted with caution.

Trials after introspective-report One key question is whether consistently reporting about distraction, counterintuitively, causes participants to be more vulnerable to attention capture (Moher, Abrams, Egeth, Yantis, \& Stuphorn, 2011; Moher \& Egeth, 2012; Tsal \& Makovski, 2006). To assess this possibility, we compared singleton-presence costs on search-only trials as a function of whether the previous trial was a searchonly trial or an introspective-report trial. We found larger singleton-presence costs on trials following introspectivereport trials $(68 \mathrm{~ms})$ than trials following search-only trials (48 ms), $t(31)=2.26, p=.031, d=0.40$. This is consistent with the interpretation that participants were more vulnerable to capture after making an introspective judgment about capture.

\section{Discussion}

In Experiment 1, participants performed an additional singleton paradigm in which they attempted to ignore a salient color singleton. On a subset of trials, participants attempted to classify whether or not they were captured by the salient distractor during the search task ("capture" vs. "no capture"). Participants received no direct feedback from the experimenter about their performance. Nonetheless, mean RT and error rates were higher on "capture" report trials than "no capture" report trials. These results are consistent with the interpretation that participants were immediately aware of attention capture when it occurred.

\section{Experiment 2}

In Experiment 1, participants could make overt eye movements. Therefore, it is unclear whether participants were detecting a covert shift of attention to the salient distractor or whether they merely had proprioceptive awareness of overt eye movements (X. Wang, Zhang, Cohen, \& Goldberg, 2007). Experiment 2 used identical methods to Experiment 1, except that eye movements were prohibited. Thus, participants had to specifically detect covert shifts of visual attention to the salient item. The predictions are identical to Experiment 1: If participants have introspective awareness of attention capture, there should be search performance costs (i.e., slower mean RT and higher error rates) on "capture" report trials compared with 
"no capture" report trials. However, if participants lack awareness of attention capture, performance on the search task should be identical on "capture" report and "no capture" report trials.

\section{Method}

All methods were identical to Experiment 1, except as follows. First, we collected a new sample of 32 participants. One participant was replaced for having a mean RT greater than 2.5 standard deviations above the group mean (greater than $880 \mathrm{~ms}$ ). Two participants were replaced for reporting "no capture" on more than $70 \%$ of introspective-report trials. Finally, two participants were replaced for having eye movements on more than $30 \%$ of trials. Of the final sample of 32 participants, 16 were women and 16 were men. The mean age was 19.6 years.

Second, an eye-tracker camera was used to detect trials with eye movements. An SR Research EyeLink1000+ deskmounted system recorded eye position monocularly from the right eye at $500 \mathrm{~Hz}$. Participants initiated each trial by maintaining gaze position on within $1.5^{\circ}$ of the fixation cross for $500 \mathrm{~ms}$. Once this fixation requirement was met, the search array appeared until response. If gaze shifted more than $1.5^{\circ}$ from the center of the screen, the trial was immediately terminated and a warning display ("Moved Eyes!" in white Arial font) was presented with a $200-\mathrm{Hz}$ tone for $500 \mathrm{~ms}$. Participants were also warned on time-out trials (i.e., when RT was greater than 2,000 ms). Both time-out trials and trials with eye movements were removed from all analyses.

Each search item was located $3.0^{\circ}$ from fixation (just as in Experiment 1). Therefore, our requirement of maintaining gaze position within $1.5^{\circ}$ of central fixation should be sufficient to prevent saccades to search items. At the beginning of each block, the eye tracker was calibrated using a 9point calibration technique. To initiate a trial, a participant first was required to make a central fixation for $500 \mathrm{~ms}$. If participants failed to do this for more than 8 seconds, we recalibrated the eye tracker before continuing.

\section{Results}

We used the same criteria to exclude trials as Experiment 1. Trials with abnormal RTs ( $0.9 \%$ of trials) were excluded from all analyses. Inaccurate responses $(4.0 \%)$ were excluded from RT analyses. We also excluded trials with eye movements (14.3\% of trials) from all analyses. Mean RTs for searchonly and introspective-report trials are depicted in Fig. 3.

Search-only trials As shown in Fig. 3, participants were slower on singleton-present trials $(658 \mathrm{~ms})$ than singleton-absent trials (622 ms): a 36-ms singleton presence cost, $t(31)=7.79, p<$ $.001, d=1.38$. This result indicates that participants were captured by the singleton-present trials, replicating Experiment 1 and previous studies using the version of the additional singleton paradigm that encourages singleton detection mode (Bacon \& Egeth, 1994; Theeuwes, 1992). Error rates were similar on singleton-present trials $(4.6 \%)$ and singleton-absent trials $(4.1 \%), t(31)=1.01, p=.319, d=0.18$.

Introspective-report trials As in Experiment 1, we compared manual RT on trials where participants reported "capture" or "no capture" by the color singleton. Participants were slower to detect the target on "capture" report trials $(690 \mathrm{~ms})$ than "no capture" report trials $(636 \mathrm{~ms}), t(31)=6.35, p<.001, d=1.12$. Note that 28 out of 32 participants showed this basic pattern of results, with slower RT on "capture" report trials compared with "no capture" report trials.

Participants also had higher error rates on "capture" report trials $(6.2 \%)$ than "no capture" report trials $(1.4 \%), t(31)=$ $5.63, p<.001, d=1.00$. Altogether, this pattern of results suggests that participants had some level of awareness of visual distraction, even without feedback from the experimenter. These results replicate Experiment 1 but demonstrate that the basic pattern of results is not due to the overt eye movements.

If participants had perfect accuracy at classifying whether they were captured, performance on "no capture" report trials should be functionally equivalent to trials where the singleton was absent. However, responses were slightly slower on "no capture" trials $(636 \mathrm{~ms})$ than in singleton-absent trials $(622$ $\mathrm{ms}), t(31)=2.72, p=.011, d=0.48$. This indicates that participants could not classify whether they were captured with perfect accuracy, although it should be noted that the difference between "no capture" report trials and singletonabsent trials is numerically small (17 ms).

Post-experiment surveys As in Experiment 1, participants completed a brief questionnaire after the search task, where they indicated their overall level of distraction by the singleton

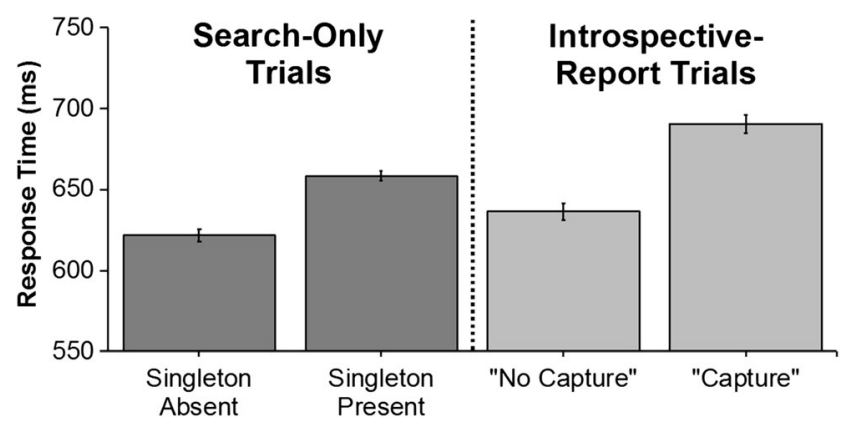

Fig. 3 Mean response times from Experiment 2 on search-only trials and introspective-report trials. The singleton was always present on introspective-report trials. Trials where participants reported distraction by the salient item are labeled "capture" trials, and trials where participants reported no distraction by the salient item are labeled "no capture" trials 
( $1=$ not at all; $5=$ very high $)$. On average, participants reported a distraction level of 3.1 . We then correlated each individual's score on the survey with their singleton-presence cost on search-only trials. Participants who reported higher levels of distraction did not have higher singleton-presence costs $(r=$ $-.05, p=.795)$. This result contrasts with the correlation results of Experiment 1 (which showed a modest correlation of $r$ $=.43$ ) and demonstrates the unreliability of questionnaire approaches to metacognition (Burra \& Kerzel, 2014; Proulx, 2011). Although participants demonstrated an ability to immediately classify whether or not they were captured, participants were not able to estimate their overall level of capture at the end of the experiment. This may be due to failures of longterm memory or an inability to accurately estimate overall probabilities of capture in comparison to other individuals.

We also correlated the percentage of "capture" report trials for each participant with responses on the post-experiment survey and singleton-presence costs on search-only trials. The percentage of "capture" report trials was uncorrelated with the magnitude of singleton-presence costs on search only trials $(r=.16, p=.223)$, and the post-experiment survey score $(r=.22, p=.219)$. As stated in Experiment 1, we explicitly encouraged participants to report "capture" and "no capture" on a roughly equal number of trials and these correlations should therefore be interpreted with caution.

Trials after introspective-report To further examine the possibility that attention capture increased as a result of the introspective-report trials, we again compared singletonpresence costs on search-only trials as a function of the previous trial type (search-only or introspective report). There was a nonsignificant trend for higher singleton-presence costs for trials following introspective-report trials $(47 \mathrm{~ms})$ than trials following search-only trials (34 ms), $t(31)=1.66, p=.107, d=$ 0.29 .

\section{Discussion}

Experiment 2 was identical to Experiment 1, except that participants were not allowed to make overt eye movements. Importantly, on introspective-report trials, both mean RT and error rates on the search task were higher on "capture" report trials than "no capture" report trials. This indicates that participants had some level of introspective awareness of visual distraction when it occurred. This internal awareness cannot be explained as mere proprioception of overt eye movements, which were prohibited and discarded from all analyses.

\section{Experiment 3}

In Experiments 1 and 2, manual RTs and error rates were both higher on "capture" report trials than "no capture" report trials.
This is consistent with the interpretation that, on "capture" report trials, participants internally detected covert shifts of attention to the salient distractor. This covert shift of attention to the singleton distractor impaired subsequent detection of the target stimulus. Neither experiment, however, provided direct evidence that attention shifted to the singleton on "capture" report trials. Therefore, it is possible that participants were detecting some other cognitive process than attention capture, such as slowed manual response or slowing of a postperceptual stage of processing (Huang, Holcombe, \& Pashler, 2004; Pashler, 1984).

Experiment 3 addressed this issue by adapting introspective-report trials to use the capture-probe technique, which provides a more direct measure of attentional allocation (Gaspelin et al., 2015). As depicted in Fig. 4, participants performed the same basic search task as Experiment 1, except that participants reported the location of a dot inside the target stimulus (left vs. right). On introspective-report trials, the search array appeared briefly with letters superimposed on each search item and then quickly disappeared. Participants were asked to report as many letters as possible. After the probe task, participants were asked to classify whether they were captured by the color singleton distractor.

If attention is allocated to a given location, participants should be more likely to report the probe letter at this location than probe letters at unattended locations (Gaspelin et al., 2015; Gaspelin \& Luck, 2018a, 2018b; Zhang, Gaspelin, \& Carlisle, 2019). Therefore, if participants are aware of attention capture, they should be more likely to report the probe letter at the singleton-distractor location on "capture" report trials than "no capture" report trials. Alternatively, if participants have no awareness of capture, probe report accuracy for the singleton distractor should be similar on "capture" and "no capture" report trials.

\section{Method}

The methods were identical to Experiment 1, except as follows. First, a new sample of 32 participants were recruited from Binghamton University, State University of New York. One participant was replaced for mean RTs exceeding 2.5 standard deviations above the mean. One participant was excluded for mean accuracy percentages less than 2.5 standard deviations below the mean. One participant was replaced for reporting "no capture" on more than $70 \%$ of trials. In the final sample of 32 participants, 18 were women and 14 were men. The mean age was 19.5 years.

Second, we adapted the task for the letter-probe technique (see Fig. 4; Gaspelin et al., 2015; Gaspelin \& Luck, 2018a, 2018b). On all trials, the tilted lines inside the shapes were removed. Instead, a small black dot that subtended $0.1^{\circ} \times 0.1^{\circ}$ appeared on either the left or right side of the shape. On search-only trials, participants made a speeded response to 


\section{Search-Only Trials}

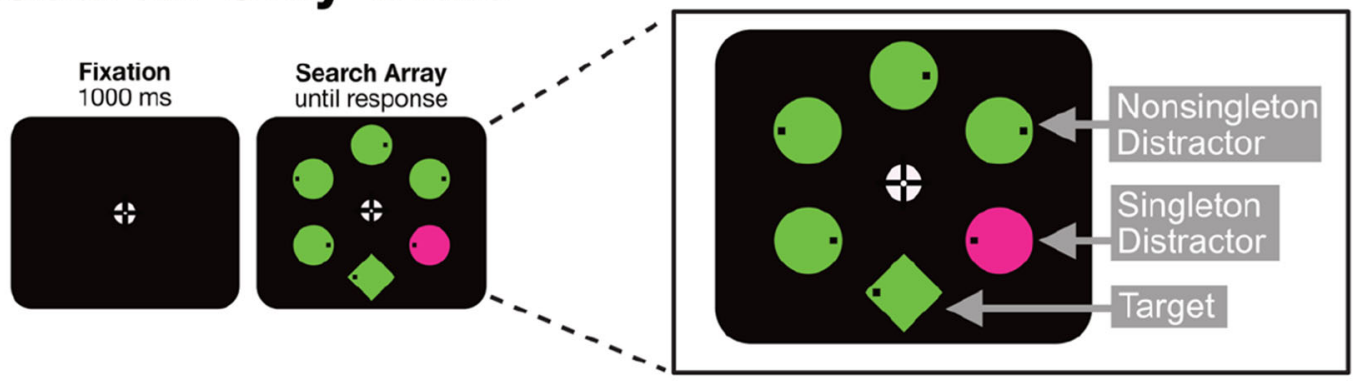

\section{Introspective-Report Trials}
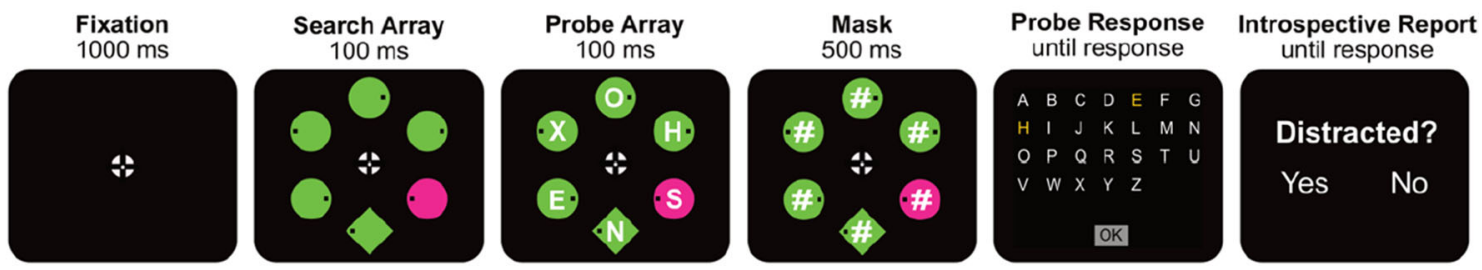

Time

Fig. 4 The stimuli and procedure from Experiment 3. On search-only trials $(75 \%)$, participants searched for a target shape and made a speeded button press regarding the location of a small black dot. On introspectivereport trials $(25 \%)$, participants first performed a capture-probe task (Gaspelin et al., 2015). Letters appeared briefly, superimposed on search

the location of the dot by clicking the left or right button of a computer mouse. On introspective-report trials, the search shapes appeared briefly for $100 \mathrm{~ms}$, and then letters were superimposed on each shape for $100 \mathrm{~ms}$. The letter at each potential search location was selected at random (from all 26 letters of the English alphabet), with the exception that no letters could repeat within a given display. The letters were then replaced by a 500-ms pattern mask (“\#”). Next, a response screen appeared and participants attempted to recall as many letters as possible. Letters were selected via mouseclick (turning them yellow) and could be unselected by clicking them again (turning them white). Participants could select anywhere between zero and six letters, and they had an unlimited time to select these letters. After the probe task, there was an introspective-report screen ("Distracted? Yes/ No"). Participants used the mouse to click "yes" or "no." As in Experiments 1 and 2, participants received both written and verbal instructions that the introspective-report prompt was specifically a question about whether attention shifted to the uniquely colored item.

\section{Results}

We used the same RT and accuracy exclusion criteria from Experiments 1 and 2 on the search-only trials. In the final analysis, $0.4 \%$ of trials were excluded for abnormal RTs and $3.4 \%$ of trials were excluded for inaccurate responses. items. Participants were then asked to recall as many letters as possible. Afterward, participants indicated whether or not they were distracted by the salient distractor. As in Experiments 1 and 2, the singleton was always present on these trials

Search-only trials Mean RT was slower in singleton-present trials $(573 \mathrm{~ms})$ than singleton-absent trials $(514 \mathrm{~ms})$ : a $59-\mathrm{ms}$ singleton presence cost $t(31)=8.25, p<.001, d=1.46$. Error rates were also higher on singleton-present trials $(3.8 \%)$ than singleton-absent trials $(3.0 \%), t(31)=2.02, p=.053, d=0.36$. Altogether, these results suggest that participants were generally distracted by the color singleton distractor, replicating Experiments 1 and 2.

Introspective-report trials Participants reported an average of 3.4 letters per trial, of which $59 \%$ of these letters were present in the probe array.

As depicted in Fig. 5a, probe report accuracy was calculated as the probability of correctly reporting probe letters at each type of search item: target, singleton distractor, and nonsingleton distractor. Note that there were four nonsingleton distractors in each display, whereas there was only one target and one singleton distractor. Therefore, to obtain a per item estimate of probe accuracy, we divided probe accuracy for the nonsingleton probes by four to obtain the average percentage of probe recall (see also Gaspelin et al., 2015; Gaspelin \& Luck, 2018b). Probe report accuracy was calculated separately for "capture" and "no capture" trials.

We first assessed probe report accuracy for singleton distractors as a function of whether participants reported "capture" or "no capture." As can be seen in Fig. 5a, probe report accuracy for letters at the singleton distractor was higher on 


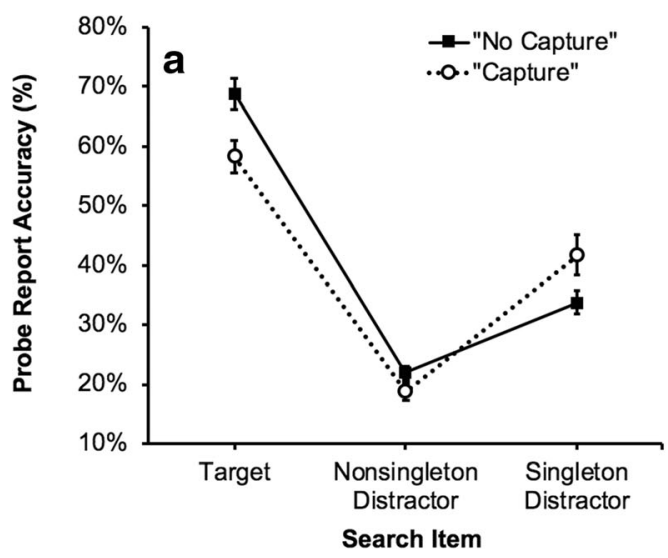

Fig. 5 a Percentage of probe report letters recalled for each type of search item on introspective-report trials in Experiment 3. Each line depicts trials where participants either reported "capture" or "no capture" by the salient item. b Mean probe capture effects for introspective-report trials where

trials where participants reported "capture" $(41.7 \%)$ than trials on which they reported "no capture" $(33.8 \%), t(31)=2.90, p=$ $.007, d=0.51$. This $8 \%$ difference suggests that covert attention was more likely to shift to the singleton distractor on "capture" trials than "no capture" trials. This is consistent with the idea that participants have some level of introspective awareness about whether visual attention was mistakenly directed to the singleton distractor.

A more direct method for assessing attention capture on probe trials is to calculate a probe capture effect (see Gaspelin et al., 2015; Gaspelin \& Luck, 2018b). This is a difference score of probe report accuracy at singleton distractor minus probe report accuracy at the average nonsingleton distractor. In other words, the probability of reporting the salient distractor item is compared with the baseline probability of reporting a nonsalient distractor item. A positive value indicates that the salient item captured attention, whereas a negative value indicates that the salient item was suppressed (e.g., see Gaspelin et al., 2015). We calculated this probe capture effect separately for introspective-report trials where participants reported "capture" and "no capture." As shown in Fig. 5b, the probe capture effect was much larger on trials where participants reported "capture" (22.9\%) than trials where they reported "no capture" $(11.9 \%), t(31)=3.79$, $p<.001, d=0.67$. This directly demonstrates that capture was in fact stronger on trials where participants reported that they were captured.

We also assessed probe report accuracy for the target as a function of whether participants reported capture. As shown in Fig. 5a, probe report accuracy for letters at the target location was much lower on "capture" report trials $(58.3 \%)$ than "no capture" report trials $(68.8 \%), t(31)=-4.11, p<.001, d=$ -0.73 . This is also consistent with the interpretation that, on trials where attention was misdirected toward the singleton distractor, attention was less likely to be directed to the target stimulus.

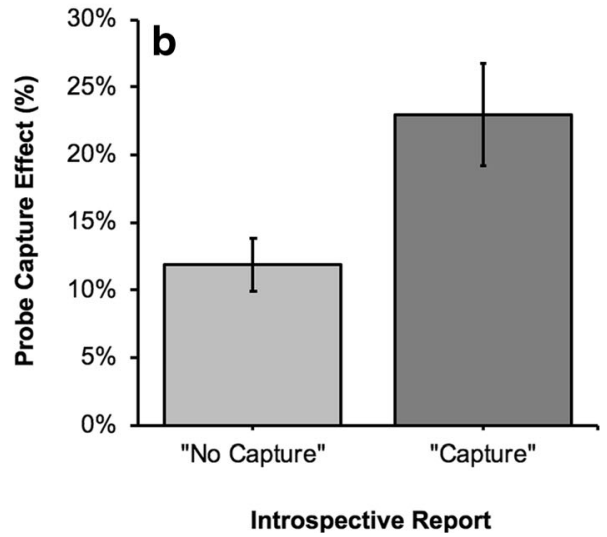

participants reported "capture" or "no capture" by the salient item in Experiment 3. Probe capture effects were calculated as probe report accuracy for the singleton item minus probe report accuracy for the nonsingleton distractor.

If participants were merely detecting general lapses in attention (i.e., "zoning out"), they should have correctly reported fewer letters on "capture" report trials than "no capture" report trials. We pooled the overall probe accuracy across search items by calculating the number of letters correctly reported, ignoring search item. There was no overall difference in probe report accuracy on "capture" report trials $(59.3 \%)$ and "no capture" report trials $(59.6 \%), t(31)=0.32$, $p=.748$.

Post-experiment surveys After completing the experiment, participants estimated their overall level of capture by the singleton with a questionnaire $(1=$ not distracting and $5=$ very distracting). The average response was 3.1 indicating that participants felt moderately distracted. We computed a correlation between scores on the survey and singleton-presence costs on search-only trials. There was no correlation between a participant's overall survey score and their singletonpresence cost $(r=.01, p=.939)$. These results support recent criticisms of questionnaire-based approaches to metacognition. In this experiment, there was direct evidence that participants were immediately aware of attention capture by the singleton on the letter-probe task. However, these questionnaire results clearly suggest that they were not able to accurately estimate their overall of level of visual distraction in comparison with other participants.

We also correlated the percentage of "capture" report trials for each participant with responses on the post-experiment survey and singleton-presence costs on search-only trials. The percentage of "capture" report trials was uncorrelated with the magnitude of singleton-presence costs on search only trials $(r=-.05, p=.791)$, and the post-experiment survey score $(r=-.06, p=.730)$. Because we explicitly encouraged participants to report "capture" and "no capture" on a roughly equal number of trials, the correlations should be interpreted with caution. 
Trials after introspective report As in Experiments 1 and 2, we again compared singleton-presence costs on search-only trials as a function of the previous trial type (search-only or introspective-report). As in Experiment 1, we found significantly higher singleton-presence costs for trials after previous introspective-report trials $(101 \mathrm{~ms})$ than trials following search-only trials (46 ms), $t(31)=7.02, p<.001, d=1.24$. This clearly suggests that after probe trials, participants were momentarily susceptible to distraction by the salient color singleton. One possibility is that reporting about attention capture made them subsequently vulnerable to attention capture (a "white bear" effect; Tsal \& Makovski, 2006).

\section{Discussion}

In Experiment 3, we used a capture-probe technique to directly assess whether visual attention shifted to the singleton on "capture" report trials (Gaspelin et al., 2015). On introspective-report trials, letters briefly appeared at each search location and then disappeared. Next, participants attempted to recall as many letters as possible. Participants should be more likely to report the letter presented at an attended location than letters at unattended locations. Thus, if participants have awareness of capture, they should be more likely to recall the probe letter at the singleton location on trials where they report "capture" than trials where they report "no capture." Indeed, there was higher singleton probe report accuracy on "capture" report trials, providing clear evidence that covert attention did shift to the singleton when participants reported visual distraction.

\section{General discussion}

The current study examined whether individuals have some level of awareness of attention capture by salient stimuli. This question is important to understanding how individuals learn to avoid attention capture, especially in real-world circumstances without performance feedback. If observers can selfmonitor distraction, there is the opportunity for them to strategically adjust top-down control to prevent future distraction. This may partially explain how individuals learn to inhibit salient items during visual search (Gaspelin, Gaspar, \& Luck, 2019; Gaspelin \& Luck, 2018b; Stilwell et al., 2019; Vatterott \& Vecera, 2012; Won \& Geng, 2018; Won, Kosoyan, $\&$ Geng, 2019). However, it is unclear if people have the ability to internally detect attention capture. Some studies have answered related questions about awareness and attention capture, but none have directly assessed whether participants had immediate awareness of whether attention shifted to a salient distractor item.

In Experiment 1, participants performed a modified additional singleton paradigm. On most trials, they searched for a target shape (e.g., diamond) among a homogenous set of distractors (e.g., circles) and attempted to ignore a salient distractor item. On a subset of trials, they immediately classified whether they were captured by the salient distractor during the preceding search task. Both mean RT and manual error rates were higher on trials where participants reported "capture" than trials where participants reported "no capture." This is consistent with the interpretation that participants were immediately aware of visual distraction when it occurred. Experiment 2 replicated this basic pattern of results, even when participants were not allowed to make overt eye movements, suggesting that participants could specifically detect covert shifts of attention to the salient distractor.

Experiment 3 provided more direct evidence that visual attention shifted to the singleton distractor on trials where participants reported "capture" by using a capture-probe technique (Gaspelin et al., 2015). Participants performed the same basic task as Experiment 1 and 2, except that the introspective-report trials were adapted to include a probe task. On these trials, letters were briefly superimposed on search item and then disappeared. Participants attempted to recall as many letters as possible. Next, participants reported whether or not they were captured by the singleton during the probe task. Importantly, we found that probe report accuracy for letters at the singleton location was higher on "capture" report trials than "no capture" report trials. Similarly, the probe capture effect (a difference score that specifically assesses attention capture by the salient item; Gaspelin et al., 2015) was nearly doubled in magnitude on trials where participants reported "capture." This again suggests that participants have some level of immediate awareness of attention capture when it occurs.

Across the experiments, there were unreliable correlations between participants' responses on a post-experiment questionnaire about distractibility and their actual performance on the attention capture task. In fact, only in Experiment 1 did we find a weakly significant correlation. This issue with delayed reports has been highlighted in the recent literature on metacognition (Fleming \& Lau, 2014), and replicates previous studies using similar approaches (Burra \& Kerzel, 2014; Proulx, 2011). The failure to estimate overall level of distraction in post-experiment questionnaires could be due to two different types of cognitive errors. First, participants may have immediate awareness of attention capture, but may forget these instances of capture by the end of the experiment due to failures of long-term memory. This could be due to either a failure to initially store memories during the task or a failure to retrieve memories of task performance during the questionnaire. A further problem with the questionnaire approach is that participants may remember instances of attention capture, but they may be unaware of how their general level of distractibility compares with that of other participants in the study. In other words, participants may have an immediate metacognitive experience of capture, but they may not have 
metacognitive knowledge of how their general distractibility compares to other individuals (Flavell, 1979). Thus, the questionnaire approach may be effective at assessing metacognitive awareness of differences in capture that occur between conditions in a within-subjects design, because participants would be able to compare their own distraction in one experimental condition to their own level of distraction in another experimental condition.

The current results suggest that participants had some level of awareness of capture, but that this awareness was imperfect. For example, in both Experiment 1 and 2, participants were significantly slower on "no capture" report trials than singleton-absent trials. If participants had perfect awareness of capture, RTs on "no capture" report trials should be functionally equivalent to trials where the singleton was physically absent. Similarly, in Experiment 3, participants reported the letter at the singleton distractor even on trials where they reported "no capture" by the singleton. If participants had perfect awareness of capture, they should have reported "no capture" only on trials where they did not report the singleton letter. Therefore, future studies are needed to more precisely estimate the level of metacognitive awareness that participants have regarding attention capture.

Relatedly, although the current study suggests that participants can have awareness of attention capture, this does not necessarily mean that participants will always have awareness of attention capture. In the current study, participants were strongly encouraged to cognitively monitor their performance. But during many instances of visual search, participants may not bother to track the location of prior attentional shifts, especially in difficult search tasks that require multiple attentional shifts (Vö et al., 2016). This would fit well with Wolfe's (1999) notion of inattentional amnesia, which suggests that memories of visual search performance might not persist as enduring representations (see also Horowitz \& Wolfe, 1998).

It is possible that participants were only indirectly aware of capture via some other cognitive process. For example, in Experiments 1 and 2, participants may have merely detected the overall speed of their manual response, which happened to be correlated with whether or not they were captured. Experiment 3 provided more direct evidence that attention shifted to the singleton on "capture" report trials. But it is possible that participants used the conscious report of probe letters to infer whether capture occurred. It is important to note that participants did not receive any feedback about performance on the probe task. Therefore, some kind of metacognitive awareness was needed in order to know that the recalled letter was from a singleton distractor. But internal awareness of what was attended may be different than metacognitive awareness of knowing where spatial attention shifted. Therefore, future research is needed to further investigate what cognitive processes participants are internally aware of while performing an attention capture task.
It remains to be seen whether metacognitive awareness could be used as an effective tool to train individuals to avoid visual distraction. Previous research has made it abundantly clear that individuals can learn to avoid attention capture by salient items (Anderson et al., 2011; Gaspelin et al., 2019; Gaspelin et al., 2015; Gaspelin \& Luck, 2018b; Leber \& Egeth, 2006; Stilwell et al., 2019; Vatterott \& Vecera, 2012; B. Wang \& Theeuwes, 2018; Won et al., 2019). But it is unclear how individuals learn to do this, especially in the absence of direct feedback about performance. On the one hand, it seems plausible that if participants can become aware of distraction when it occurs, there is the opportunity for them to adjust their attentional control settings to avoid subsequent distraction by salient stimuli (Bacon \& Egeth, 1994; Folk et al., 1992). Alternatively, it is also possible that asking participants to explicitly monitor performance may disrupt the automatic processes that are typically involved in attentional guidance. Thus, metacognitive awareness of attention capture could (ironically) lead to enhanced capture by the to-beignored salient stimuli (Cunningham \& Egeth, 2016; Moher \& Egeth, 2012). This will be an important issue for future research, especially considering recent discussions about how implicit learning may play an important role in attention capture (Awh et al., 2012; Theeuwes, 2018).

In conclusion, the present study demonstrates that observers have some level of immediate awareness of attention capture, at least under certain circumstances. This could be a valuable framework for understanding how individuals learn to avoid distraction in real-world situations where they do not receive direct feedback about performance.

Acknowledgements We thank Steven Luck and Eric Ruthruff for their helpful comments on this project.

Open practices statement The stimulus presentation programs, original data, and data summaries are available on the Open Sciences Framework (https://osf.io/kqhgf/?view_only=c8e1fa1a16f6403f96c83c25abd2d521).

\section{References}

Anderson, B. A. (2013). A value-driven mechanism of attentional selection. Journal of Vision, 13(3), 1-16. https://doi.org/10.1167/13.3.7

Anderson, B. A., \& Kim, H. (2018). Mechanisms of value-learning in the guidance of spatial attention. Cognition, 178, 26-36. https://doi.org/ 10.1016/j.cognition.2018.05.005

Anderson, B. A., Laurent, P. A., \& Yantis, S. (2011). Value-driven attentional capture. Proceedings of the National Academy of Sciences, 108(25), 10367-10371. https://doi.org/10.1073/pnas.1104047108

Awh, E., Belopolsky, A. V., \& Theeuwes, J. (2012). Top-down versus bottom-up attentional control: A failed theoretical dichotomy. Trends in Cognitive Sciences, 16(8), 437-443. https://doi.org/10. 1016/j.tics.2012.06.010

Bacon, W. F., \& Egeth, H. E. (1994). Overriding stimulus-driven attentional capture. Perception \& Psychophysics, 55(5), 485-496. https:// doi.org/10.3758/bf03205306 
Brainard, D. H. (1997). The Psychophysics Toolbox. Spatial Vision, 10(4), 433-436. https://doi.org/10.1163/156856897X00357

Burra, N., \& Kerzel, D. (2014). The distractor positivity (Pd) signals lowering of attentional priority: Evidence from event-related potentials and individual differences: Pd and lowering of attentional priority. Psychophysiology, 51(7), 685-696. https://doi.org/10.1111/ psyp. 12215

Cousineau, D. (2005). Confidence intervals in within-subject designs: A simpler solution to Loftus and Masson's method. Tutorials in Quantitative Methods for Psychology, 1(1), 42-45. https://doi.org/ 10.20982/tqmp.01.1.p042

Cunningham, C. A., \& Egeth, H. E. (2016). Taming the white bear: Initial costs and eventual benefits of distractor inhibition. Psychological Science, 27(4), 476-485. https://doi.org/10.1177/ 0956797615626564

Flavell, J. H. (1979). Metacognition and cognitive monitoring: A new area of cognitive-developmental inquiry. American Psychologist, 34(10), 906-911. https://doi.org/10.1037/0003-066X.34.10.906

Fleming, S. M., \& Lau, H. C. (2014). How to measure metacognition. Frontiers in Human Neuroscience, 8. https://doi.org/10.3389/ fnhum.2014.00443

Fletcher, L., \& Carruthers, P. (2012). Metacognition and reasoning. Philosophical Transactions of the Royal Society B: Biological Sciences, 367(1594), 1366-1378. https://doi.org/10.1098/rstb. 2011.0413

Folk, C. L., Leber, A. B., \& Egeth, H. E. (2002). Made you blink! Contingent attentional capture produces a spatial blink. Perception \& Psychophysics, 64(5), 741-753. https://doi.org/10.3758/ BF03194741

Folk, C. L., Remington, R. W., \& Johnston, J. C. (1992). Involuntary covert orienting is contingent on attentional control settings. Journal of Experimental Psychology: Human Perception and Performance, 18(4), 1030-1044. https://doi.org/10.1037//00961523.18.4.1030

Gaspar, J. M., \& McDonald, J. J. (2014). Suppression of salient objects prevents distraction in visual search. Journal of Neuroscience, 34(16), 5658-5666. https://doi.org/10.1523/JNEUROSCI.4161-13. 2014

Gaspar, J. M., \& McDonald, J. J. (2018). High level of trait anxiety leads to salience-driven distraction and compensation. Psychological Science, 29(12), 2020-2030. https://doi.org/10.1177/ 0956797618807166

Gaspelin, N., Gaspar, J. M., \& Luck, S. J. (2019). Oculomotor inhibition of salient distractors: Voluntary inhibition cannot override selection history. Visual Cognition, 27(3/4), 227-246. https://doi.org/10. 1080/13506285.2019.1600090

Gaspelin, N., Leonard, C. J., \& Luck, S. J. (2015). Direct evidence for active suppression of salient-but-irrelevant sensory inputs. Psychological Science, 26(11), 1740-1750. https://doi.org/10. $1177 / 0956797615597913$

Gaspelin, N., Leonard, C. J., \& Luck, S. J. (2017). Suppression of overt attentional capture by salient-but-irrelevant color singletons. Attention, Perception, \& Psychophysics, 79(1), 45-62. https://doi. org/10.3758/s13414-016-1209-1

Gaspelin, N., \& Luck, S. J. (2018a). Combined electrophysiological and behavioral evidence for the suppression of salient distractors. Journal of Cognitive Neuroscience, 30(9), 1265-1280. https://doi. org/10.1162/jocn_a_01279

Gaspelin, N., \& Luck, S. J. (2018b). Distinguishing among potential mechanisms of singleton suppression. Journal of Experimental Psychology: Human Perception and Performance, 44(4), 626644. https://doi.org/10.1037/xhp0000484

Gaspelin, N., \& Luck, S. J. (2018c). The role of inhibition in avoiding distraction by salient stimuli. Trends in Cognitive Sciences, 22(1), 79-92. https://doi.org/10.1016/j.tics.2017.11.001
Gaspelin, N., \& Luck, S. J. (2019). Inhibition as a potential resolution of the attentional capture debate. Current Opinion in Psychology, 29, 12-18. https://doi.org/10.1016/j.copsyc.2018.10.013

Henderson, J. (2003). Human gaze control during real-world scene perception. Trends in Cognitive Sciences, 7(11), 498-504. https://doi. org/10.1016/j.tics.2003.09.006

Horowitz, T. S., \& Wolfe, J. M. (1998). Visual search has no memory. Nature, 394(6), 575-77. https://doi.org/10.1038/29068

Horowitz, T. S., Wolfe, J. M., Alvarez, G. A., Cohen, M. A., \& Kuzmova, Y. I. (2009). The speed of free will. Quarterly Journal of Experimental Psychology, 62(11), 2262-2288. https://doi.org/10. 1080/17470210902732155

Huang, L., Holcombe, A. O., \& Pashler, H. (2004). Repetition priming in visual search: Episodic retrieval, not feature priming. Memory \& Cognition, 32(1), 12-20. https://doi.org/10.3758/BF03195816

Lamy, D., Alon, L., Carmel, T., \& Shalev, N. (2015). The role of conscious perception in attentional capture and object-file updating. Psychological Science, 26(1), 48-57. https://doi.org/10.1177/ 0956797614556777

Leber, A. B., \& Egeth, H. E. (2006). It's under control: Top-down search strategies can override attentional capture. Psychonomic Bulletin \& Review 13(1), 132-38. https://doi.org/10.3758/BF03193824

Maniscalco, B., \& Lau, H. (2012). A signal detection theoretic approach for estimating metacognitive sensitivity from confidence ratings. Consciousness and Cognition, 21(1), 422-430. https://doi.org/10. 1016/j.concog.2011.09.021

Moher, J., Abrams, J., Egeth, H. E., Yantis, S., \& Stuphorn, V. (2011). Trial-by-trial adjustments of top-down set modulate oculomotor capture. Psychonomic Bulletin \& Review, 18(5), 897-903. https:// doi.org/10.3758/s13423-011-0118-5

Moher, J., \& Egeth, H. E. (2012). The ignoring paradox: Cueing distractor features leads first to selection, then to inhibition of tobe-ignored items. Attention, Perception, \& Psychophysics, 74(8), 1590-1605. https://doi.org/10.3758/s13414-012-0358-0

Morey, R. D. (2008). Confidence intervals from normalized data: A correction to Cousineau (2005). Tutorial in Quantitative Methods for Psychology, 4(2), 61-64. https://doi.org/10.20982/tqmp.04.2.p061

Mulckhuyse, M., Talsma, D., \& Theeuwes, J. (2007). Grabbing attention without knowing: Automatic capture of attention by subliminal spatial cues. Visual Cognition, 15(7), 779-788. https://doi.org/10.1080/ 13506280701307001

Nisbett, R. E., \& Wilson, T. D. (1977). Telling more than we can know: Verbal reports on mental processes. Psychological Review, 84(3), 231-259. https://doi.org/10.1037/0033-295X.84.3.231

Pashler, H. (1984). Processing stages in overlapping tasks: Evidence for a central bottleneck. Journal of Experimental Psychology: Human Perception and Performance. 10(3), 358-377. https://doi.org/10. 1037/0096-1523.10.3.358

Pashler, H. (1988). Familiarity and visual change detection. Perception \& Psychophysics, 44(4), 369-378. https://doi.org/10.3758/ BF03210419

Proulx, M. J. (2011). Individual differences and metacognitive knowledge of visual search strategy. PLOS ONE, 6(10), e27043. https:// doi.org/10.1371/journal.pone.0027043

Stilwell, B. T., Bahle, B., \& Vecera, S. P. (2019). Feature-based statistical regularities of distractors modulate attentional capture. Journal of Experimental Psychology: Human Perception and Performance, 45(3), 419-433. https://doi.org/10.1037/xhp0000613

Theeuwes, J. (1992). Perceptual selectivity for color and form. Perception \& Psychophysics, 51(6), 599-606. https://doi.org/10.3758/ BF03211656

Theeuwes, J. (2018). Visual selection: Usually fast and automatic; seldom slow and volitional. Journal of Cognition, 1, 1-15. https://doi.org/ 10.5334/joc. 13

Tsal, Y., \& Makovski, T. (2006). The attentional white bear phenomenon: The mandatory allocation of attention to expected distractor 
locations. Journal of Experimental Psychology: Human Perception and Performance, 32(2), 351-363. https://doi.org/10.1037/00961523.32.2.351

Vatterott, D. B., \& Vecera, S. P. (2012). Experience-dependent attentional tuning of distractor rejection. Psychonomic Bulletin \& Review, 19(5), 871-878. https://doi.org/10.3758/s13423-012-0280-4

Vö, M. L.-H., Aizenman, A. M., \& Wolfe, J. M. (2016). You think you know where you looked? You better look again. Journal of Experimental Psychology: Human Perception and Performance, 42(10), 1477-1481. https://doi.org/10.1037/xhp0000264

Wang, B., \& Theeuwes, J. (2018). Statistical regularities modulate attentional capture. Journal of Experimental Psychology: Human Perception \& Performance, 44(1), 13-17. https://doi.org/10.1037/ xhp0000472

Wang, X., Zhang, M., Cohen, I. S., \& Goldberg, M. E. (2007). The proprioceptive representation of eye position in monkey primary somatosensory cortex. Nature Neuroscience, 10(5), 640-646. https://doi.org/10.1038/nn1878

Wolfe, J. M (1999). Inattentional amnesia. In V. Coltheart (Ed.), Fleeting memories (pp. 71-94). Cambridge, MA: MIT Press. https://doi.org/ $10.1037 / \mathrm{e} 536982012-171$
Wolfe, J. M., \& Horowitz, T. S. (2017). Five factors that guide attention in visual search. Nature Human Behaviour, 1(3), 1-8. https://doi.org/ $10.1038 / \mathrm{s} 41562-017-0058$

Won, B.-Y., \& Geng, J. J. (2018). Learned suppression for multiple distractors in visual search. Journal of Experimental Psychology: Human Perception and Performance, 44(7), 1128-1141. https:// doi.org/10.1037/xhp0000521

Won, B., Kosoyan, M., Geng, J.J. (2019). Evidence for second-order singleton suppression based on probabilistic expectations. Journal of Experimental Psychology: Human Perception and Performance, 45(1), 125-138. https://doi.org/10.1037/xhp0000594

Zhang, Z., Gaspelin, N., Carlisle, N.B. (2019). Probing early attention following negative and positive templates. Attention, Perception, \& Psychophysics. Advance online publication. https://doi.org/10. 3758/s13414-019-01864-8

Publisher's note Springer Nature remains neutral with regard to jurisdictional claims in published maps and institutional affiliations. 